\title{
Influence of the final drive ratio, electric motor size and battery capacity on fuel consumption of a parallel plug-in hybrid electric vehicle
}

\author{
O. Torres ${ }^{1}$, B. Bader ${ }^{1}$, J. L. Romeral ${ }^{1,3}$, G. Lux $^{2}$ \& J. A. Ortega ${ }^{1}$ \\ ${ }^{1}$ MCIA Innovation Electronics, \\ Technical University of Catalonia (UPC), Spain \\ ${ }^{2}$ SEAT Technical Centre, Spain \\ ${ }^{3}$ CTM Technical Centre, Spain
}

\begin{abstract}
The purpose of this paper is to study the influence of the electric motor (EM) size, final drive ratio (FD) and the battery capacity (BAT) of a parallel plug-in hybrid electric vehicle (PHEV) regarding fuel consumption. The energy efficiency of a certain vehicle drivetrain depends on the size of the components. For this reason and for cost reasons it is necessary to study the optimal dimensions of the components that minimize the fuel consumption for a given driving cycle. In this publication the influence of the size of the electric motor, final drive ratio and also the battery capacity are analysed using the Response Surface Methodology (RSM) of the Design of Experiments (DoE) technique. A parallel PHEV has been parameterized and simulated to obtain the fuel consumption over NEDC driving cycle using Modelica/Dymola. This paper contains an introduction, a brief explanation of the modelled parallel HEV, a description of the all electric range operating strategy based on rules, an explanation of the RSM method, the simulation results, and finally the conclusions of this study.
\end{abstract}

Keywords: optimal, sizing, models, simulation, PHEV, fuel, electric, consumption. 


\section{Introduction}

In a HEV there are a lot of components that affect directly the fuel consumption [1]. There are studies in the current literature about how to get the optimal components minimizing the fuel consumption using different techniques. The proposal of this paper is to apply DoE to know the influence of the components on the fuel consumption. In order to obtain it, firstly a parallel PHEV concept with a rule-based operating strategy is modelled using Modelica/Dymola [2]. This model is used to evaluate the fuel consumption using different simulation experiments changing the size of the components. In this case study, the influence of the electric motor size, final drive ratio and the size of the battery capacity has been studied. A rule-based operating strategy (All Electric Range strategy) was implemented which selects the driving mode depending on the driver requests giving inputs to the electric motor (EM), internal combustion engine (ICE), gearbox and to the clutch. Then a Design of Experiments (DoE) parameterization using the Response Surface Methodology (RSM) is evaluated. The NEDC driving cycle is used in this model in order to calculate the fuel consumption for each experiment changing the size of the EM, the ratio of the final drive and also the capacity of the battery. Once the experiments are done a second order model fitting is used to calculate the coefficients of the equation terms to know the curvature and the tendency of the fuel consumption for the components studied. After that the minimum of this model equation is computed in order to calculate the optimal size of the components that minimize the fuel consumption for this case study.

\section{Parallel PHEV model}

The parallel vehicle model done is based on a systematic approach using energetic models for different subsystems. The internal combustion engine is modelled using a look-up-table for the fuel consumption, while for the electric motor a loss map is used. Also basic vehicle subsystems such as driver, chassis, clutch, gearbox, and forces calculation are modelled [3, 4]. Models of the battery, battery management system, inverter, and control units are also implemented.

The parallel hybrid configuration switches between the two power sources, the internal combustion engine and the electric motor. Depending on the situation, both power sources can also be used simultaneously to achieve maximum power output. Figure 1 shows the system configuration of a parallel PHEV. The advantage of this vehicle structure is that the system has the ability to offer high efficiency during highway driving conditions avoiding low efficient points of the ICE. On the other hand, the electric motor can be used during urban driving cycles to prevent the ICE from operating in its low-efficiency range, thus providing higher overall efficiency. 


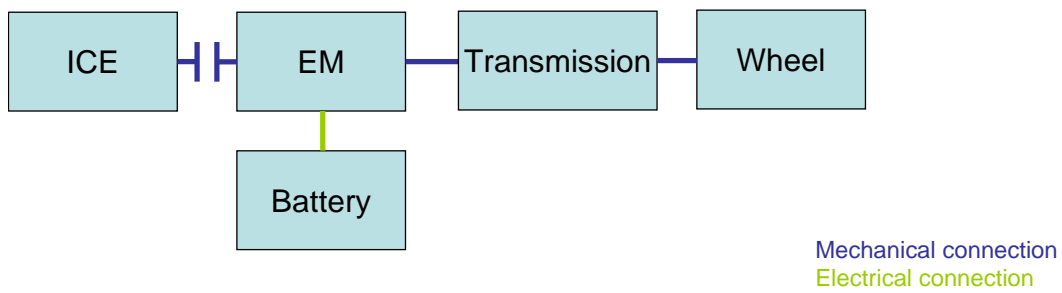

Figure 1: $\quad$ Powertrain of parallel PHEV model.

\section{All electric range operating strategy}

To simulate the fuel consumption using this vehicle model under the NEDC driving cycle, a basic operating strategy was implemented in the hybrid control unit model. The All Electric Range (AER) operating strategy based on rules has two different modes called charge depleting (CD) and charge sustaining (CS). For each mode (figure 2) five operating modes are implemented (figure 3). The CD mode uses the energy stored in the battery until to reach a minimum SOC (State Of Charge) of $20 \%$ using electric and regeneration modes. Afterwards in CS part the strategy attempts to maintain this minimum level SOC mode using also recharge, boost and ICE driving mode. At the time that the battery SOC reaches his minimum, the strategy enters the CS mode, in which the combustion engine is also used.

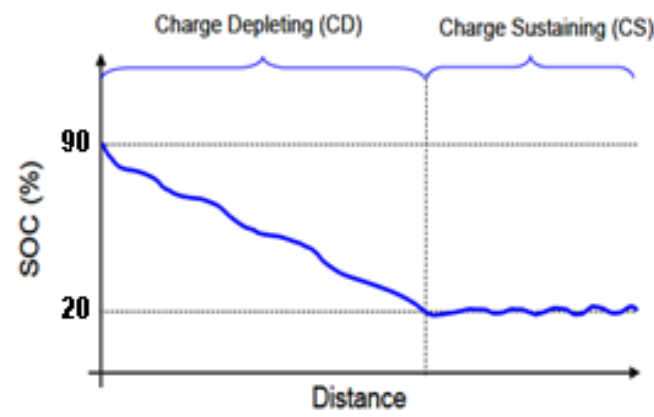

Figure 2: $\quad$ All Electric range operating strategy.

Depending on the vehicle speed, in this case at $50 \mathrm{~km} / \mathrm{h}$, or also on the E-drive if is intentionally applied by the driver due to is a PHEV, the vehicle enters the hybrid mode (Boost or Charge) or remains in electric mode. The strategy has to choose the mode of operation, the gear and torque set point of the engines. The ICE and the EM, in a parallel hybrid electric vehicle, work on the same mechanical axis to add their torque. The maximum torque is the sum of the curves of maximum torque of EM and ICE. When power demand is low, it may be sufficient to use only the EM ("Electric mode"). In regenerative braking the 

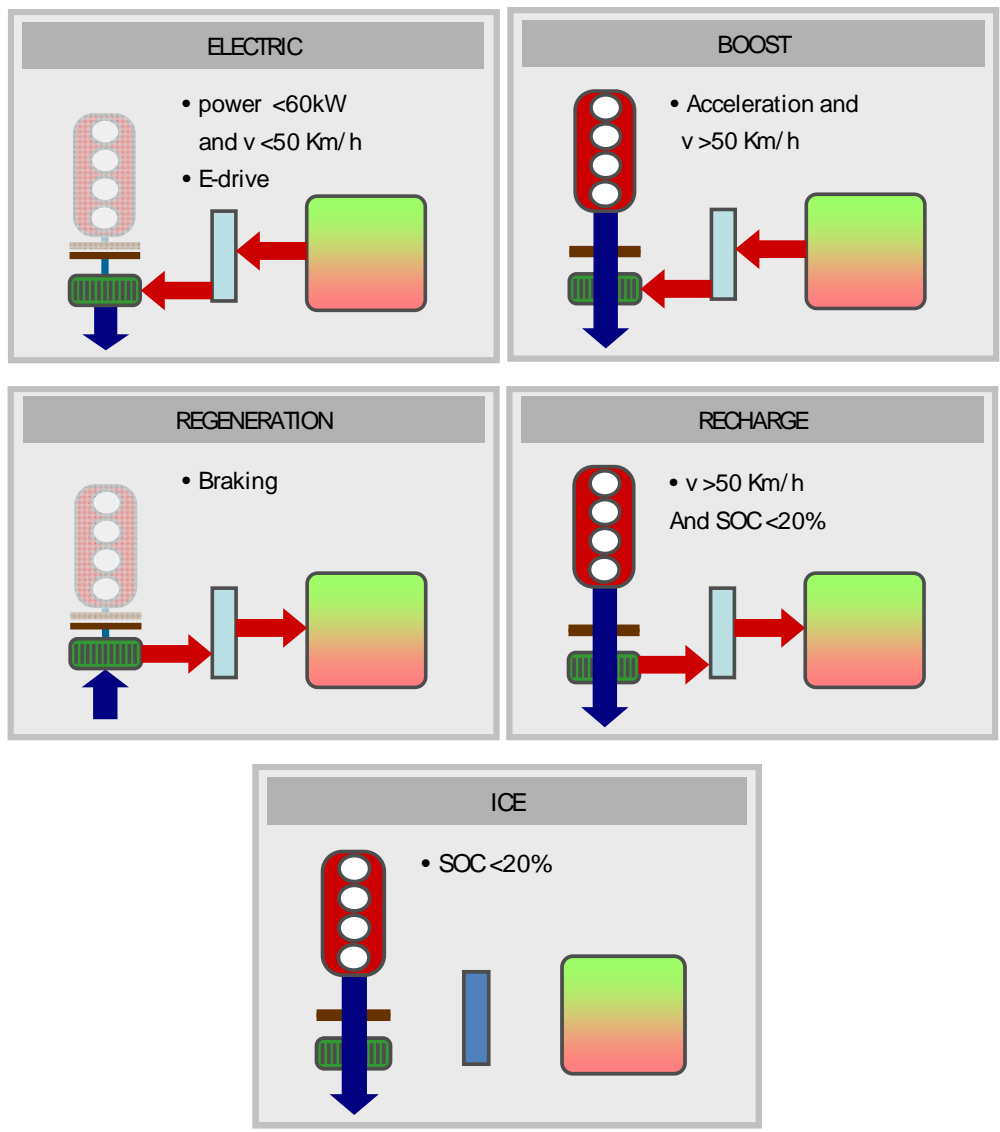

Figure 3: $\quad$ Operating modes of parallel HEV model.

electric motor is used as a generator charging the battery when the SOC does not exceed its maximum value, which means that the battery can store this energy ("Regeneration mode"). In hybrid mode has been implemented "Recharge mode" and "Boost mode", where in both the traction motors are used. In "Recharge mode" mode, combustion engine generates more torque demanded by the driver to recharge the battery. If the driver, demands more power than the electric motor can generate, "Boost mode" is entered, in which the two machines accelerate the vehicle. Finally the "ICE mode" is used when the SOC is too low and the ICE power is directly transferred to the wheel. The changing process between the modes depends on the SOC of the battery. To avoid continuous changes between the modes, a hysteresis of $5 \mathrm{~km} / \mathrm{h}$ has included, in case that the vehicle drives just at this edge. At speeds above $50 \mathrm{~km} / \mathrm{h}$ the engine is turned on and the vehicle enters to the hybrid mode. Therefore if the vehicle speed exceeds the limit of the maximum speed in electric mode, the car changes into the hybrid mode. This means that the ICE is on and the two machines contribute to the 
acceleration of the vehicle. The strategy intends to use the ICE at every moment at the highest efficiency point of the current angular velocity. If the driver request more torque, the strategy sends the maximum possible torque, getting out of this efficient curve.

The gear is chosen by the vehicle through the Hybrid Control Unit subsystem and the driver has no chance of selection, as in many conventional vehicles with automatic transmission, and is determined according to the speed. Moreover, the decision of the gear follows the same strategy in hybrid mode as in electric mode. The shift points are adapted while driving at low speed to the EM and at high speed to the ICE, as this engine has to always work in his optimum zone.

\section{Design of experiments' parameterization}

In order to evaluate the influence of the EM size, final drive ratio and battery capacity a DoE parameterization is applied using Matlab/Simulink. The concept of DoE uses a set of experiments which has to be performed by the experimenter (see figure 4). The aim of this so-called design is to parameterize a process or system by performing each experiment and to draw conclusions about the significant behaviour of the studied object from the results of the experiments.

A full factorial design with three factors and three levels for each factor is created in this case study. This means that the DoE design matrix is formed by twenty-seven experiments ( 3 factors $\wedge 3$ levels $=27$ experiments). A full factorial design means to test all the experiments of the region. In this case it is possible to calculate all experiments because the simulation run time, and the number of experiments are reasonable to be calculated in less than 200h approximately. Also to use a full factorial design has the advantage to increase the accuracy of

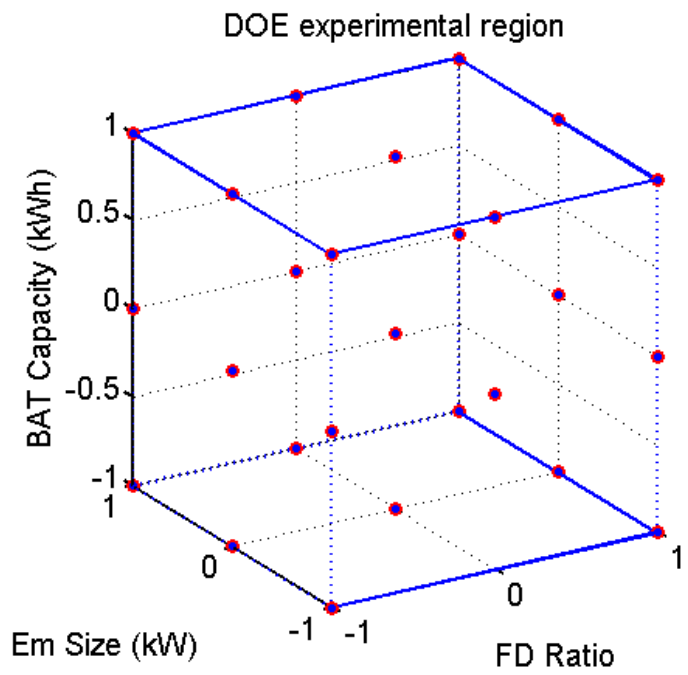

Figure 4: $\quad$ Experimental region of the Design of Experiments. 
the results. These experiments are different options for the size and possible combinations of the EM size, final drive ratio and battery capacity. As each experiment to simulate does not require a lot of computation time, and now only three factors are selected to study, a full factorial design is chosen. Each row of such a design contains a combination of the values of those variables that are changed within their limits of variation. In the context under consideration here, each of these input-variable combinations is used for parameterisation of a simulation run, in that the values from the respective input variable combination are used to parameterize the size of the EM, the final drive ratio and the battery capacity of the vehicle incorporated in the simulation model. During a simulation run, the selected values are assigned to the variables of the experiment, and the fuel consumption and electrical autonomy under the standardized cycle (NEDC) are obtained.

\section{Response Surface Methodology}

The complexity of the hybrid drivetrains results in a large number of potential independent variables that affect the dependent variable under consideration to different extents. For this reason it is important to know how strong the effects of these factors are. A useful tool to know an optimal design for regression models, in this case the design that minimizes the fuel consumption is the RSM. The regression analysis is needed for modelling and to analyze several variables, to obtain the relationship between a dependent variable and independent variables. This means to calculate how the typical value of the dependent variable changes when any one of the independent variables is varied, while the other independent variables are held fixed. RSM is applied to the results in order to evaluate the influence of each component on the final result, in this case the fuel consumption during charge sustaining mode.

The RSM (figure 5) explores the relationships between several explanatory variables and one or more response variables. The main idea of RSM [5, 6] is to

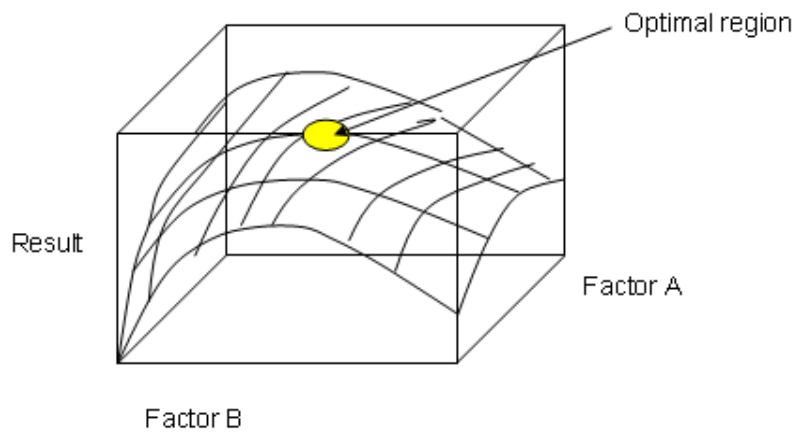

Figure 5: Example of the Response Surface Methodology concept using two factors and obtaining the optimal region. In this paper case will be the minimization of the fuel consumption. 
use a sequence of designed experiments to obtain an optimal response, the minimum of fuel consumption. Since the dependency of fuel consumption on the large number of variables does not exist as an explicit target function, they are approximated in the preceding process step of modeling, in the form of an adapted polynomial (equation 1). A second order model for the regression fitting is used to analyze the curvature and shape of the obtained model. Analyzing the surface obtained it is possible to estimate the influence of each component studied [7].

$$
Y=\beta_{0}+\sum_{i=1}^{k} \beta_{i} X_{i}+\sum_{i=1}^{k} \beta_{i i} X_{i}^{2}+\sum_{i=1}^{k-1} \sum_{j=2}^{k} \beta_{i j} X_{i} X_{j}+\varepsilon
$$

\section{Definition of the parameters for this case study}

The selected sizes of the components evaluated for each experiment are in a reasonable range, being any option as a real possible solution for a parallel HEV. The fixed size of the vehicle parameters are shown in Table 1. To size the EM, the efficiency map is used for the nominal value (see Figure 6), and an adaptation of this is done for the upper and lower size taking into account the losses in each electric motor tested (Table 2). The driving cycle used in overall simulations is the standard NEDC (Figure 7).

Table 1: $\quad$ Fixed size of the vehicle parameters.

\begin{tabular}{|c|c|}
\hline Components & Size \\
\hline ICE $(\mathrm{kW})$ & 51 \\
\hline Battery Voltage $(\mathrm{V})$ & 300 \\
\hline Weight $(\mathrm{kg})$ & $1508 / 1565 / 1630 *$ \\
\hline Af $\left(\mathrm{m}^{2}\right)$ & 2.2 \\
\hline Gears & 7 \\
\hline
\end{tabular}

*The vehicle weight changes with the battery capacity (see Table 2).

Table 2: $\quad$ Size of the powertrain variables tested.

\begin{tabular}{|c|c|c|c|}
\hline Components & Lower & Nominal & Upper \\
\hline $\begin{array}{c}\text { EM } \\
(k W)\end{array}$ & 30 & 40 & 50 \\
\hline $\begin{array}{c}\text { Battery } \\
\text { Capacity } \\
(k W h)\end{array}$ & 4 & 8 & 12 \\
\hline $\begin{array}{c}\text { Final drive } \\
\text { ratio }\end{array}$ & 3.5 & 4 & 4.5 \\
\hline
\end{tabular}




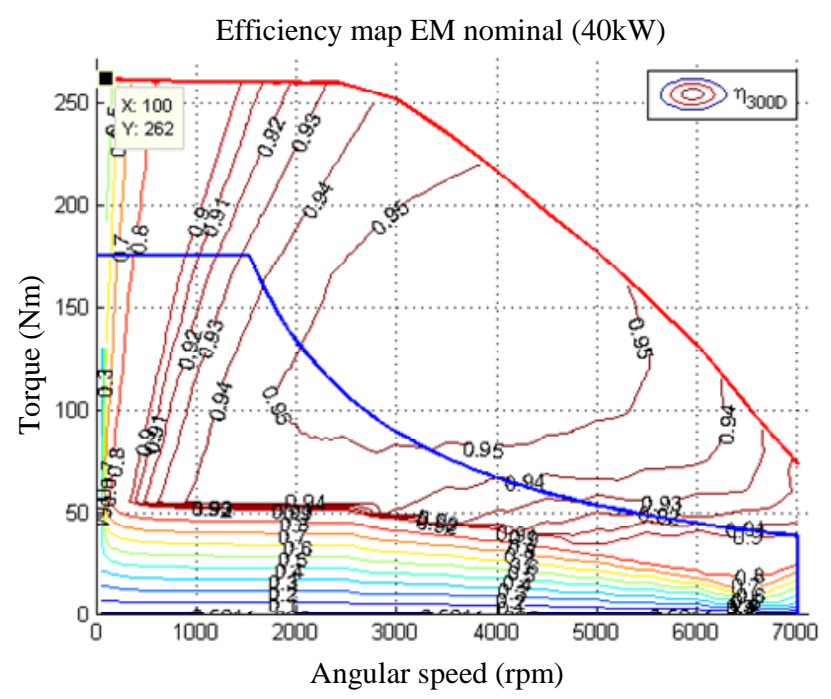

Figure 6: $\quad$ Efficiency map Electric Motor nominal.

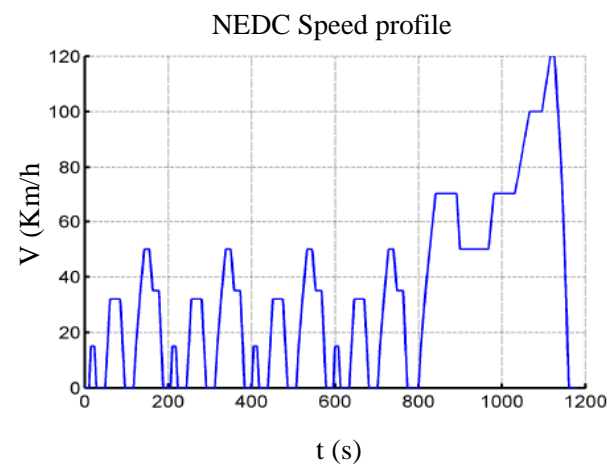

Figure 7: $\quad$ Speed profile of NEDC driving cycle.

\section{Parameterization results}

When planning an experimental program that will allow carrying out the study on the effect of factors on the response variable, the first step to take is the choice of factors that will be used in the experiment. In this case the factors selected are the EM power, the ratio of the final drive and the battery capacity. Once the factors are determined, the next step is to select the ranges of each factor to be analyzed in the experimental region selected. The experimental design matrix used is: 


$$
\begin{aligned}
& \mathrm{X}=\left[\begin{array}{lll}
-1 & -1 & -1
\end{array}\right. \\
& \begin{array}{lll}
-1 & -1 & 0
\end{array} \\
& \begin{array}{lll}
-1 & -1 & 1
\end{array} \\
& \begin{array}{lll}
-1 & 0 & -1
\end{array} \\
& \begin{array}{lll}
-1 & 0 & 0
\end{array} \\
& \begin{array}{lll}
-1 & 0 & 1
\end{array} \\
& \begin{array}{lll}
-1 & 1 & -1
\end{array} \\
& \begin{array}{lll}
-1 & 1 & 0
\end{array} \\
& \begin{array}{lll}
-1 & 1 & 1
\end{array} \\
& \begin{array}{lll}
0 & 1 & -1
\end{array} \\
& \begin{array}{lll}
0 & -1 & 0
\end{array} \\
& \begin{array}{lll}
0 & -1 & 1
\end{array} \\
& \begin{array}{lll}
0 & 0 & -1
\end{array} \\
& \begin{array}{lll}
0 & 0 & 0
\end{array} \\
& \begin{array}{lll}
0 & 0 & 1
\end{array} \\
& \begin{array}{lll}
0 & 1 & -1
\end{array} \\
& \begin{array}{lll}
0 & 1 & 0
\end{array} \\
& \begin{array}{lll}
0 & 1 & 1
\end{array} \\
& \begin{array}{lll}
1 & -1 & -1
\end{array} \\
& \begin{array}{lll}
1 & -1 & 0
\end{array} \\
& \begin{array}{lll}
1 & -1 & 1
\end{array} \\
& \begin{array}{lll}
1 & 0 & -1
\end{array} \\
& 100 \\
& \begin{array}{lll}
1 & 0 & 1
\end{array} \\
& \begin{array}{lll}
1 & 1 & -1
\end{array} \\
& \begin{array}{lll}
1 & 1 & 0
\end{array} \\
& \left.\begin{array}{lll}
1 & 1 & 1
\end{array}\right] \text {; }
\end{aligned}
$$

where each row corresponds to one experiment, first column to the final drive ratio, and second column to the electric motor size. The values are coded meaning " 1 " the lower value, " 0 " the nominal value, and " 1 " the upper value.

Once the fuel consumption and electrical autonomy are obtained, it is possible to calculate the fuel consumption according ECE-R 101, where the fuel consumption for a PHEV based on the NEDC (Figure 7) is defined as (equation (2)):

$$
F C(l / 100 \mathrm{~km})=\frac{D e \cdot F C 1+25 \mathrm{~km} \cdot F C 2}{D e+25 \mathrm{~km}}
$$

FC1 $\rightarrow$ Fuel consumption (1/100km) during charge depleting FC2 $\rightarrow$ Fuel consumption (l/100km) during charge sustaining $\mathrm{De} \rightarrow$ Electric autonomy $(\mathrm{km})$.

Using the design of the experiments performed it is very useful to obtain the response surface model fitting the data set collected at points of design to a polynomial equation. Using the data of fuel consumption simulated in all 
experiments it is possible to obtain a second-order mathematical model with terms linear, quadratic and binary interactions of the three independent variables $\left(X_{1}, X_{2}, X_{3}\right)$ analyzed (equation (3)).

$$
\begin{aligned}
& \mu_{y}=\beta_{0}+\beta_{1} x_{1}+\beta_{2} x_{2}+\beta_{3} x_{3}+\beta_{11} x_{1}^{2}+ \\
& \beta_{22} x_{2}{ }^{2}+\beta_{33} x_{3}^{2}+\beta_{12} x_{1} x_{2}+\beta_{13} x_{1} x_{3}+\beta_{23} x_{2} x_{3}+\zeta
\end{aligned}
$$

where,

$\mu_{y}=$ Average value of the response variable (fuel consumption)

$\beta_{0}=$ Average value of the response, without the effect of the factors $\left(x_{1}, \mathrm{x}_{2}\right)$

$\beta_{1}=$ Lineal effect of factor 1 on the average response

$\beta_{2}=$ Lineal effect of factor 2 on the average response

$\beta_{3}=$ Lineal effect of factor 3 on the average response

$\beta_{11}=$ Quadratic effect of factor 1 on the average response

$\beta_{22}=$ Quadratic effect of factor 2 on the average response

$\beta_{33}=$ Quadratic effect of factor 3 on the average response

$\beta_{12}=$ Interaction effect between $x_{1}, x_{2}$ on the response

$\beta_{13}=$ Interaction effect between $x_{1}, x_{3}$ on the response

$\beta_{23}=$ Interaction effect between $x_{2}, x_{3}$ on the response

$\zeta=$ error

Table 3: $\quad$ Coefficient results for second order model.

\begin{tabular}{|c|c|c|}
\hline Equation & Variables & Results \\
\hline$\beta_{0}$ & Constant & 1,6240 \\
\hline$\beta_{1}$ & $\mathrm{FD}$ & 0,0823 \\
\hline$\beta_{2}$ & $\mathrm{EM}$ & $-0,0145$ \\
\hline$\beta_{3}$ & $\mathrm{BAT}$ & $-0,516$ \\
\hline$\beta_{11}$ & $\mathrm{FD} * \mathrm{FD}$ & 0,0197 \\
\hline$\beta_{22}$ & $\mathrm{EM} * \mathrm{EM}$ & $-0,0016$ \\
\hline$\beta_{33}$ & $\mathrm{BAT}^{*} \mathrm{BAT}$ & 0,1776 \\
\hline$\beta_{12}$ & $\mathrm{FD} * \mathrm{EM}$ & 0,0076 \\
\hline$\beta_{13}$ & FD*BAT & $-0,0293$ \\
\hline$\beta_{23}$ & EM*BAT & 0,0014 \\
\hline$\zeta$ & Error & 0,042 \\
\hline
\end{tabular}


The obtained coefficients of each term are shown in table 3. Notice that the major component that contributes more to the result is the final drive ratio (FD). Therefore the regression equation for this case is exposed in equation (4):

Fuel consumption $(\mathrm{l} / 100 \mathrm{~km})=1,6240+0,0823 \cdot F D-0,0145^{*} \cdot E M-0,516 \cdot B A T$

$$
\begin{gathered}
+0,0197 \cdot F D^{2}-0,0016 \cdot E M^{2}+0,1776 \cdot B A T^{2} \\
+0,0076 \cdot(F D \cdot E M)-0,0293 \cdot(F D \cdot B A T) \\
+0,0014 \cdot(E M \cdot B A T)+0,042
\end{gathered}
$$

The equation means that the BAT capacity has an important contribution to the fuel consumption, meanwhile the FD ratio and EM size has a minor effect compared to the battery capacity.

In order to a better understanding of the equation obtained, in Figure 8 is shown the surface response of the fuel consumption for the EM size, FD ratio and BAT capacity under the analyzed ranges. The combination that minimizes the fuel consumption is with the lowest final drive ratio (3.5) and a high battery capacity $(12 \mathrm{kWh})$. The minimum of this function is $1.24 \mathrm{l} / 100 \mathrm{~km}$ of fuel consumption using the $\mathrm{FD}=3.5, \mathrm{BAT}=12 \mathrm{kWh}$ and $\mathrm{EM}=50 \mathrm{~kW}$. In Figure 9 it's shown also the influence of the components studied by fixing the EM size equal to $40 \mathrm{~kW}$.
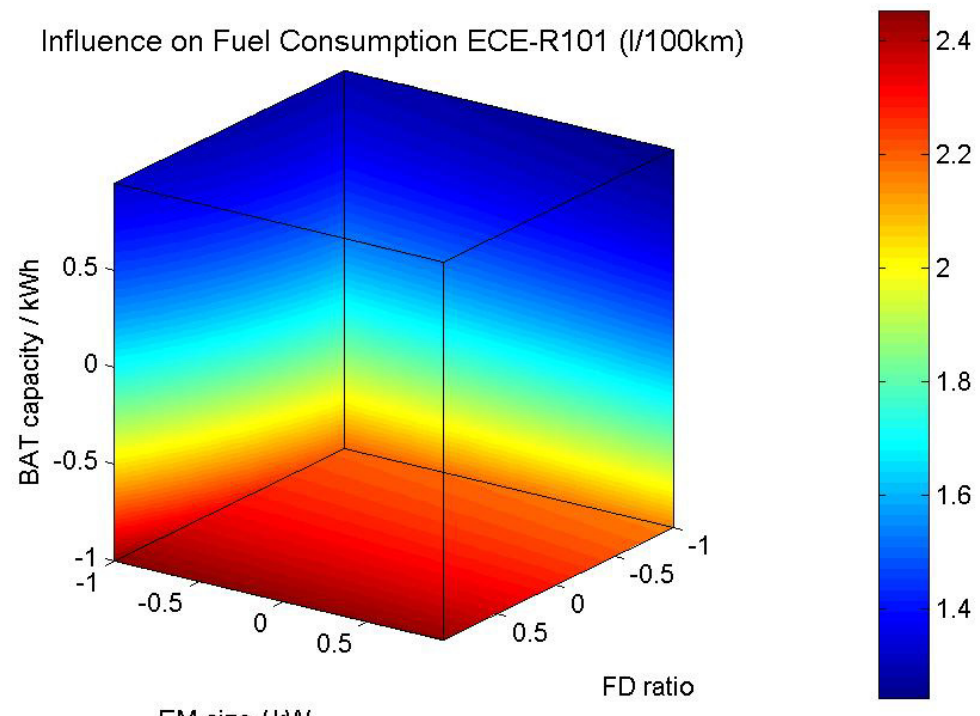

EM size / $\mathrm{kW}$

Figure 8: Response surface of fuel consumption among FD ratio, EM size and BAT capacity. 


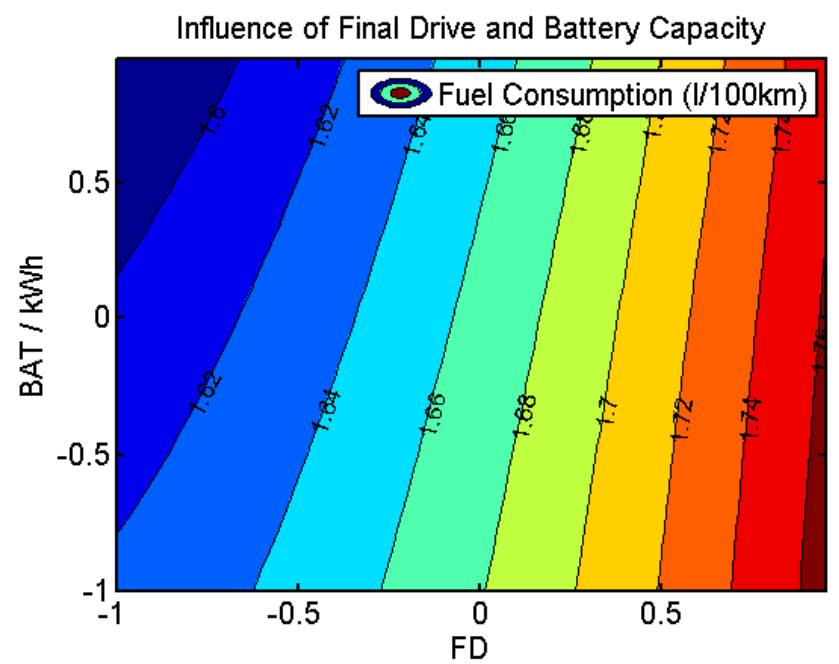

Figure 9: Influence of the FD ratio and BAT capacity to the fuel consumption fixing the EM size.

\section{Conclusions}

This study gives several conclusions and suggestions in the design process of a parallel PHEV. Design of Experiments and Response Surface Methodology has been used to analyze how the variation of parameters in the components affects on the fuel consumption. Also has been calculated a first approximation of a parameterized equation that describes the system on an analytic way regarding the fuel consumption using ECE-R101. It has been observed a strong influence of the BAT capacity below proportional, in example, doubling the electrical autonomy, the fuel consumption is reduced by less than half. The current high cost of the battery makes that the optimal set of components could be different and in future the components costs will be considered in this study. The FD ratio has a direct influence on the fuel consumption in CS mode, and indirectly through electric consumption due to the electrical autonomy increases. In future it will be studied his influence to check the maximum vehicle speed and also the acceleration performance. And finally in this case study the EM size has a little influence due to the electrical machine is always working on an optimal efficiency region, but must be taken into account in future work because it will directly also affect the acceleration performance. Therefore the 3.5 for the final drive ratio and the $12 \mathrm{kWh}$ for the battery capacity give better results considering the fuel consumption under this specific case setting.

In future studies more components will be introduced to the system analysis, in order to get more accuracy in the equation of the fuel consumption, and also the electric consumption during charge depleting mode, maximum vehicle speed, acceleration performance and the components cost will be studied, in order to create a very detailed cost function that parameterizes the parallel PHEV. 


\section{Acknowledgements}

VERDE is a CENIT project funded by the Ministry of Economy and Competitiveness of the Spanish Government. It is led by SEAT, coordinated by CTM Centre Tecnològic and integrated by the following companies: AIA, Cegasa, Circutor, Cobra, Endesa, Ficosa, Green Power, Iberdrola, Infranor, Lear, Mapro, Red Eléctrica España, Siemens, Rovalma and Tècnicas Reunidas; and Research Institutes: AICIA, ASCAMM, CIDETEC, CIRCE, CNM del CSIC, CTM Centre Tecnològic, IIC, IIT de la Universidad Pontificie de Comillas, IREC, Leitat, Tecnalia, Universitat Politècnica Catalunya (UPC) and Universidad Carlos III.

\section{References}

[1] C C Chan, S Wong, The State of the Art of Electric Vehicles Technology, Power Electronics and Motion Control Conference, 2004. IPEMC 2004

[2] Modelica ${ }^{\circledR}$ - A Unified Object-Oriented Language for Physical Systems Modeling Language Specification Version 3.0, September 5, 2007

[3] Dariusz Cieslar, Jens G. Linden, Keith J. Burnham, Matthew Hancock, Francis Assadian, Jaguar Cars and Land Rover, Modelling of Hybrid Electric Vehicle All Wheel Drive driveline system incorporating clutch models, 19th International Conference on Systems Engineering, 2008

[4] Giorgio Rizzoni, Lino Guzzella, Bernd M. Baumann, Unified Modeling of Hybrid Electric Vehicle Drivetrains, IEEE/ASME Transactions on Mechatronics, vol. 4, no. 3, September 1999

[5] André I. Khuri, Siuli Mukhopadhyay, Response surface methodology, Volume 2, March/April 2010, John Wiley \& Sons, Inc.

[6] Khuri, André I., Cornell, John A., Response Surfaces. Design and Analyse, Statistics: Textbooks and monographs. 81. Marcel Dekker, 1987. New York.

[7] Nuran Bradley, The Response Surface Methodology, Thesis, 2007 